\title{
Erratum: Precision measurements with photon-subtracted or photon-added Gaussian states [Phys. Rev. A 90, 013821 (2014)]
}

\author{
Daniel Braun, Pu Jian, Olivier Pinel, and Nicolas Treps
}

(Received 6 June 2015; published 23 June 2015)

DOI: 10.1103/PhysRevA.91.069905

PACS number(s): 42.50.St, 03.65.Ta, 42.50.Ex, 42.50.Lc, 99.10.Cd

Our formula (8) in the original paper contains a sign error in the last term: the +1 in the bracket should read $\mp 1$. The results for the photon-subtracted states are not affected, but some formulas for photon-added states are changed and partly substantially simplified:

Equation (31) should read

$$
\begin{aligned}
W(x, p)\left[\hat{\rho}^{+}\right]= & \frac{e^{-\left[(p-\bar{p})^{2} / \Gamma\right]-(x-\bar{x})^{2} \Gamma}}{\pi \Gamma^{2}\left[2\left(1+\bar{p}^{2}+\bar{x}^{2}\right)+\Gamma^{-1}+\Gamma\right]}\left[2 p^{2}+2 \bar{p}^{2}+\left(-1+4 p^{2}\right) \Gamma+2\left(-1+p^{2}+x^{2}\right) \Gamma^{2}\right. \\
& \left.+\left(-1+4 x^{2}-4 x \bar{x}\right) \Gamma^{3}+2(x-\bar{x})^{2} \Gamma^{4}-4 p \bar{p}(1+\Gamma)\right] .
\end{aligned}
$$

It is still true that the large $\bar{x}$ expansion leads to the exact same expression as for photon subtraction in Eq. (17). Equation (32) should read

$$
I\left[\hat{\rho}^{(+)}(\theta)\right]=\frac{2 \Gamma\left[3+8 \Gamma+2\left(5+4 \bar{x}^{2}+2 \bar{x}^{4}\right) \Gamma^{2}+8\left(1+\bar{x}^{2}\right) \Gamma^{3}+3 \Gamma^{4}\right] \bar{x}^{\prime 2}}{\left[1+2\left(1+\bar{x}^{2}\right) \Gamma+\Gamma^{2}\right]^{2}} .
$$

Two lines before Eq. (33), the expression $3+\bar{x}^{2}>0$ should read $1+\bar{x}^{2}>0$. The conclusion that no divergence is observed for $N \ll 1$ is unchanged. The corrected expressions for Eqs. (33) and (34) are

$$
I\left[\hat{\rho}^{(+)}(\theta)\right]=\frac{\left\{3+\Gamma\left[4 \bar{x}^{4} \Gamma+4 \bar{x}^{2}\left(3+2 \Gamma+3 \Gamma^{2}\right)+3\left(4+6 \Gamma+4 \Gamma^{2}+\Gamma^{3}\right)\right]\right\} \Gamma^{\prime 2}}{2\left[\Gamma+2\left(1+\bar{x}^{2}\right) \Gamma^{2}+\Gamma^{3}\right]^{2}},
$$

and

$$
I\left[\hat{\rho}^{(+)}(\theta)\right]=\frac{3 \Gamma^{\prime 2}}{2 \Gamma^{2}},
$$

where the latter is identical to the expression for the photon-subtracted state for all $\Gamma$. Finally, Eq. (A1) in the Appendix should be corrected to

$$
\begin{aligned}
I\left[\hat{\rho}^{(+)}(\theta)\right]= & \frac{1}{2\left[\Gamma+2\left(1+\bar{p}^{2}+\bar{x}^{2}\right) \Gamma^{2}+\Gamma^{3}\right]^{2}} 4 \Gamma\left\{3+\Gamma\left[8+8 \bar{p}^{2}+10 \Gamma+4\left(\bar{p}^{2}+\bar{x}^{2}\right)\left(2+\bar{p}^{2}+\bar{x}^{2}\right) \Gamma+8\left(1+\bar{x}^{2}\right) \Gamma^{2}+3 \Gamma^{3}\right]\right\} \\
& \times\left[\left(\bar{p}^{\prime}\right)^{2}+\Gamma^{2}\left(\bar{x}^{\prime}\right)^{2}\right]+32 \Gamma^{2}\left\{\bar{p}\left[1+\left(1+\bar{p}^{2}+\bar{x}^{2}\right) \Gamma\right] \bar{p}^{\prime}-\bar{x} \Gamma\left(1+\bar{p}^{2}+\bar{x}^{2}+\Gamma\right) \bar{x}^{\prime}\right\} \Gamma^{\prime} \\
& +\left\{3+\Gamma\left[12 \bar{p}^{2}+4\left(\bar{p}^{2}+\bar{x}^{2}\right)\left(2+\bar{p}^{2}+\bar{x}^{2}\right) \Gamma+12\left(1+\bar{p}^{2}+\bar{x}^{2}\right) \Gamma^{2}+3 \Gamma^{3}+6\left(2+2 \bar{x}^{2}+3 \Gamma\right)\right]\right\}\left(\Gamma^{\prime}\right)^{2}
\end{aligned}
$$

We thank B. Gard for pointing out the mistake in Eq. (8). 